\title{
AppliChem
}

\section{DNA decontamination: DNA-ExitusPlus in comparison with conventional reagents}

\author{
Here we present a completely new DNA decontamination reagent DNA-ExitusPlus. In comparison \\ with conventional products, DNA-ExitusPlus guarantees fast and efficient destruction of nucleic acids \\ without corrosive or toxic properties. DNA-ExitusPlus was developed by AppliChem GmbH, Darmstadt, \\ in cooperation with multiBIND biotec $\mathrm{GmbH}$, Dortmund.
}

The polymerase chain reaction (PCR) has set the standard for DNA amplification technology ${ }^{1}$, and the rapid extension of PCR technologies has resulted in multiple new applications ${ }^{2,3}$. The latest protocols and enzyme variants for PCR reactions allow the detection of even single DNA molecules ${ }^{1}$. One important consequence of the improved sensitivity in DNA amplification is the necessity to avoid any contamination from unwanted external DNA molecules.

The latest research in genetic technology has demonstrated that the presence of free DNA molecules can pose serious problems ${ }^{4,5}$. Infections, biological transformations or recombination of genomes can be generated by free DNA plasmids or fragments ${ }^{3-7}$. Changes in viral and bacterial infectivity are observed and are related to the phenomenon of resistance against multiple antibiotics ${ }^{8}$. As a result, the detection of DNA contamination or prevention of amplification artifacts in PCR expreiments is essential for all applications as well as biological containment and safety.

Investigations of the properties of conventional DNA decontamination reagents revealed two major problems. First, none of the reagents studied destroyed DNA molecules efficiently, and second, existing reagents typically contain components with corrosive or even toxic properties. As a consequence, we saw the necessity to develop new solutions for effective DNA decontamination.

DNA decontamination reagents use three different molecular principles for destruction or inactivation of genetic material: modification, denaturation and degradation. Safe DNA decontamination depends on the degradation of DNA into very small fragments. We developed a DNA degradation test to compare conventional decontamination reagents with the new DNA-ExitusPlus. This test allows sensitive quantification of the fragmentation process (Fig. 1).

\section{Karl-Heinz Esser ${ }^{1}$, Wolfram H Marx ${ }^{2}$ \& Thomas Lisowsky ${ }^{1}$}

${ }^{1}$ multiBIND biotec GmbH, Otto-Hahn-Str. 15, D-44227, Dortmund, Germany.

${ }^{2}$ AppliChem GmbH, Ottoweg 4, D-64291 Darmstadt, Germany. Correspondence should be addressed to T.L. (thomas.lisowsky@gmx.de) or W.H.M. (w.marx@applichem.de).

PUBLISHED ONLINE XX XXXXXX 2006; DOI:10.1038/NMETHXXX

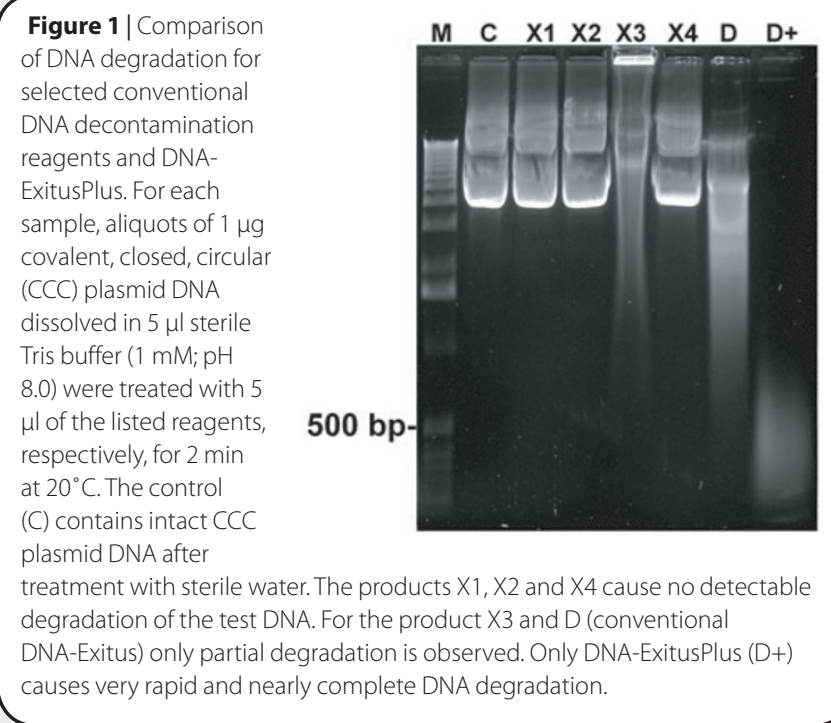

One surprising result was that some of the conventional reagents are based only on the principles of modification or denaturation. This is revealed by the complete absence of any degraded DNA molecules (Fig. 1). Thus the genetic information is only masked but not destroyed. By chemical demasking reactions the DNA sequences of these molecules would be available and active again. Based on our knowledge about gene technology and the principles of recombination, we concluded that these reagents are no longer appropriate.

However, even reagents that degrade DNA cause only limited and partial destruction. Hence, very large DNA fragments containing the complete genetic information still survive treatment. Only DNA-ExitusPlus achieves rapid and efficient degradation.

Another severe disadvantage of conventional reagents is revealed in a test of their corrosive potential. For this purpose different metal plates were incubated with aliquots of the reagents (Fig. 2). This test demonstrates that all currently available products contain aggressive chemicals with corrosive, harmful or even toxic effects. Known ingredients of conventional reagents are azides, mineral acids like 


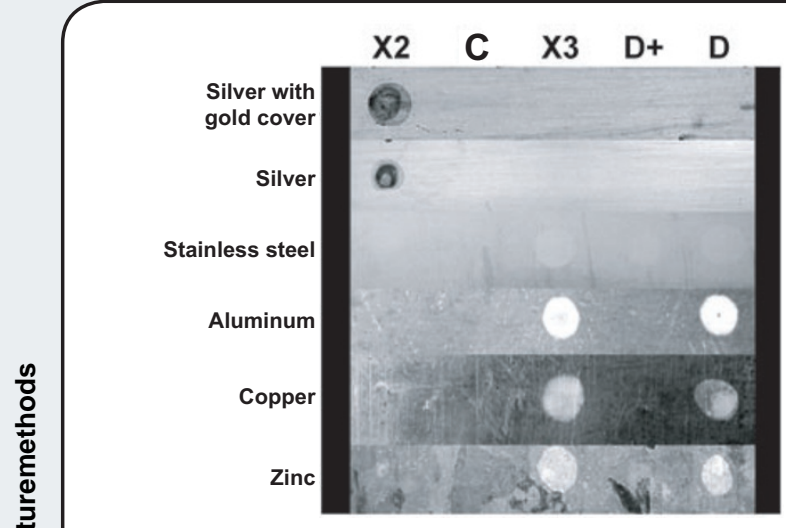

Figure $\mathbf{2}$ | The corrosive potential of selected conventional DNA decontamination reagents in comparison with DNA-ExitusPlus. Aliquots of 10 $\mu \mathrm{l}$ from each listed reagent were applied to metal plates that are typically used for laboratory materials and equipment, and then incubated for $20 \mathrm{~min}$. Sterile water was used as a control (C). The reagents $\mathrm{X} 2, \mathrm{X} 3$ and $\mathrm{D}$ cause irreversible corrosion and damage to many of the metal surfaces. For DNA-ExitusPlus (D+) no damage is observed for any of the surfaces. In some cases, one observes a polishing effect owing to the removal of dirt or oxide layers.

phosphoric acid or hydrochloric acid, aggressive peroxides or strong alkaline substances like sodium hydroxide. Therefore, after only 20 min of incubation, irreversible damage of metal surfaces is observed (Fig. 2). The newly developed reagent DNA-ExitusPlus exhibits its unique characteristics especially in this test. For all metal surfaces no corrosion is observed; DNA-ExitusPlus was also tested on many different plastic surfaces without any indication of damage (data not shown). DNA-ExitusPlus offers an efficient, gentle and environmentally safe alternative and proves its superiority to other available decontamination reagents. DNA-ExitusPlus not only degrades and removes all DNA molecules with high efficiency but is neither toxic nor corrosive.

In summary, one observes the following new and unique characteristics: (i) catalytic and cooperative effects guarantee rapid nonenzymatic degradation of nucleic acids, (ii) all components of DNA-ExitusPlus are biodegradable and not harmful or toxic, (iii) no aggressive mineral acids or alkaline substances are used. Equipment and materials are not damaged or corroded even after prolonged incubation

Currently, the most effective method for decontamination appears to be autoclaving. Under standard conditions for autoclaving, DNA molecules are degraded into fragments of 20 to 30 base pairs. However, recent investigations with highly sensitive PCR analysis demonstrate that even after autoclaving larger DNA fragments can persist ${ }^{9}$. Furthermore, autoclaving can only be used for decontamination of heat-resistant materials and equipment that fit into the autoclave. Decontamination of laboratory benches or larger equipment is impossible.

Efficient degradation of DNA molecules by DNA-ExitusPlus was monitored by PCR analysis (Fig. 3) proving that no amplifiable DNA templates are present. Today, only very different nonstandardized PCR tests are used as controls for successful DNA decontamination. In the case of large DNA control templates, low DNA concentrations and high dilutions in the washing steps, evidence for successful DNA decontamination is very limited. Therefore one has to be very cautious

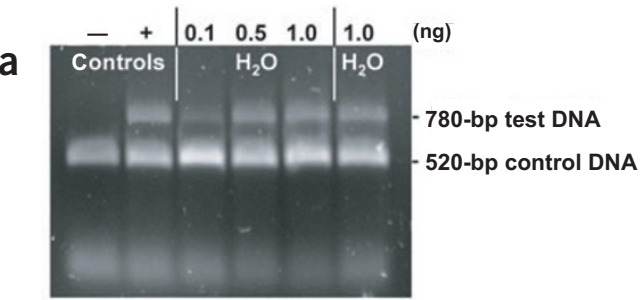

b

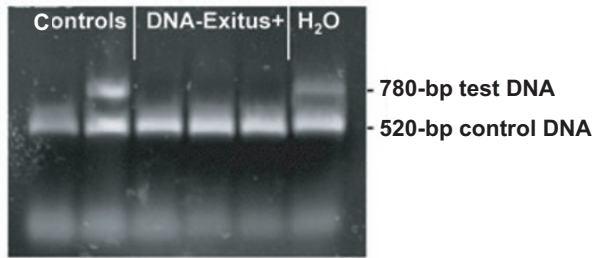

Figure $\mathbf{3} \mid P C R$ test for the complete removal of DNA contamination by DNA-ExitusPlus. $(\mathbf{a}, \mathbf{b})$ Selected amounts of test DNA were lyophilized on the inner surface of PCR tubes, after which the samples were incubated for $20 \mathrm{~s}$ with either sterile water (a) or DNA-ExitusPlus (b). For the PCR test reaction, mixtures of $50 \mu \mathrm{l}$ were added to each tube. This reaction mixture contains primers for the amplification of control and test DNA. The control DNA is added to each sample and shows that the PCR reaction is not inhibited. The negative control (with sterile water) exhibits DNA bands for the test and control templates. The PCR reaction after treatment with DNA-ExitusPlus amplifies the control DNA only.

about using a single PCR test as evidence for complete decontamination because such a PCR test would also be negative in the case where DNA is only modified or masked. For complete evaluation of the potential of a DNA decontamination reagent one has to use PCR analysis in combination with a sensitive DNA degradation test.

The tests described here reveal the unique characteristics of DNA-ExitusPlus. These properties offer new opportunities for potential applications in the health sector, the life sciences, medical hygiene, food production and the household. We are convinced that this product defines a new standard for efficient, rapid and gentle DNA decontamination. According to the latest results on biological activities of free DNA molecules, such a product is critical for the new tasks concerning biological containment and safety.

1. Innis, M.A. et al. (eds.) PCR Protocols: A Guide to Methods and Applications (Academic Press, Inc., San Diego, 1990).

2. Cavalli-Sforza, L.L. The Human Genome Diversity Project: past, present and future. Nat. Rev. Genet. 6, 333-340 (2005).

3. Tumpey, T.M. et al. Characterization of the reconstructed 1918 Spanish influenza pandemic virus. Science 310, 77-80 (2005).

4. Burns, P.A. et al. Transformation of mouse skin endothelial cells in vivo by direct application of plasmid DNA encoding the human T24 $\mathrm{H}$-ras oncogene. Oncogene 6, 1973-1978 (1991).

5. Moniz, M. et al. HPV DNA vaccines. Front. Biosci. 8, d55-d68 (2003).

6. Gibbs, M.J. et al. Recombination in the hemagglutinin gene of the 1918 "Spanish flu." Science 293, 1842-1845 (2001).

7. Kaiser, J. Biocontainment. 1918 flu experiments spark concerns about biosafety. Science 306, 591 (2004).

8. Guyot, A. et al. Molecular epidemiology of multi-resistant Escherichia coli. J. Hosp. Infect. 43, 39-48 (1999).

9. Elhafi, G. et al. Microwave or autoclave treatments destroy the infectivity of infectious bronchitis virus and avian pneumovirus but allow detection by reverse transcriptase-polymerase chain reaction. Avian Pathol. 33, 303-306 (2004).

This article was submitted to Nature Methods by a commercial organization and has not been peer reviewed. Nature Methods takes no responsibility for the accuracy or otherwise of the information provided. 